\title{
SENSITIVITY ANALYSIS OF SOLUTIONS FOR A SYSTEM OF GENERALIZED PARAMETRIC NONLINEAR QUASIVARIATIONAL INEQUALITIES
}

\author{
ZEQING LIU, BEIBEI ZHU, SHIN MIN KANG, AND GWANG IL KIM
}

Received 28 December 2004

A new class of system of generalized parametric nonlinear quasivariational inequalities involving various classes of mappings is introduced and studied. With the properties of maximal monotone mappings, the equivalence between the class of system of generalized parametric nonlinear quasivariational inequalities and a class of fixed point problems is proved and an iterative algorithm with errors is constructed. A few existence and uniqueness results and sensitivity analysis of solutions are also established for the system of generalized nonlinear parametric quasivariational inequalities and some convergence results of iterative sequence generated by the algorithm with errors are proved.

\section{Introduction}

Recently, variational inequalities constitute an important modelling tool in pure and applied mathematics. In 1996, Zhu and Marcotte [28] introduced and investigated a class of system of variational inequalities in $R^{n}$. Afterwards, Nie et al. [21], Verma [22, 23, 24, 25, 26], Wu et al. [27], and others studied the approximation and solvability of a few kinds of various systems of variational inequalities in Hilbert spaces. Moreover, Agarwal et al. [1], Dafermos [3], Dong et al. [4], and Liu et al. [20], and others considered the sensitivity of solutions for several kinds of parametric variational inequalities in Hilbert spaces.

Motivated and inspired by the research work $[1,2,3,4,5,6,7,8,9,10,11,12,13$, $14,15,16,17,18,19,20,21,22,23,24,25,26,27,28]$, in this paper, we introduce and study a new class of system of generalized parametric nonlinear quasivariational inequalities involving various classes of mappings in Hilbert spaces. Using some properties of the maximal monotone mapping, we prove the equivalence between the class of system of generalized parametric nonlinear quasivariational inequalities and a class of fixed point problems and also construct an iterative algorithm with errors for solving the system of generalized parametric nonlinear quasivariational inequalities. We also establish a few existence and uniqueness results as well as the sensitivity analysis of solutions for the system of generalized nonlinear parametric quasivariational inequalities, and prove some convergence results of iterative sequence generated by the algorithm with errors. The results presented in this paper extend, improve, and unify some known results in $[21,26,27]$ and others. 
1796 Generalized parametric nonlinear quasivariational inequalities

\section{Preliminaries}

Let $H$ be a real Hilbert space with an inner product $\langle\cdot, \cdot\rangle$ and norm $\|\cdot\|$, respectively. Let $K$ be a nonempty closed convex subset of $H$ and let $G$ be an open subset of $H$ in which the parameter $\lambda$ takes values. Suppose that $M: H \times G \rightarrow 2^{H}$ is such that for each $\lambda \in G$, $M(\cdot, \lambda): H \rightarrow 2^{H}$ is maximal monotone. Let $S, T: H \times G \rightarrow H$ be any mappings, let $\rho$ and $\beta$ be positive constants, and let $f$ and $g$ be arbitrary elements in $H$. For each $\lambda \in G$, we consider the following problem: find $x, y \in H$ such that

$$
\begin{aligned}
& 0 \in \rho(S(y, \lambda)-T(y, \lambda)-f)+x-y+\rho M(x, \lambda), \\
& 0 \in \beta(S(x, \lambda)-T(x, \lambda)-g)+y-x+\beta M(y, \lambda),
\end{aligned}
$$

which is known as the system of generalized parametric nonlinear quasivariational inequalities.

If $S(x, \lambda)=x, T(x, \lambda)=T(x)$, and $M(x, \lambda)=\partial \varphi(x)$ for any $(x, \lambda) \in H \times G$, where $\partial \varphi$ denotes the subdifferential of a proper, convex, and lower semicontinuous functional $\varphi: H \rightarrow R \cup\{+\infty\}$, then the problem (2.1) reduces to the following problem: determine elements $x, y \in H$ such that

$$
\begin{gathered}
\langle\rho(S(y)-T(y)-f)+x-y, u-x\rangle \geq \rho \varphi(x)-\rho \varphi(u), \\
\langle\beta(S(x)-T(x)-g)+y-x, u-y\rangle \geq \beta \varphi(y)-\beta \varphi(u) \quad \forall u \in H,
\end{gathered}
$$

which is said to be the system of generalized nonlinear variational inequalities studied by Nie et al. in [21].

In case $\varphi=\delta_{K}$, where $\delta_{K}$ denotes the indicator function of the nonempty closed convex subset $K$ of $H$, then the problem (2.2) reduces to the following problem:

$$
\begin{gathered}
\langle\rho(S(y)-T(y)-f)+x-y, u-x\rangle \geq 0, \\
\langle\beta(S(x)-T(x)-g)+y-x, u-y\rangle \geq 0 \quad \forall u \in K,
\end{gathered}
$$

which was introduced and studied by Wu et al. in [27].

If $f=g=T=0$, then the problem (2.3) is equivalent to finding $x, y \in K$ such that

$$
\begin{gathered}
\langle\rho S(y)+x-y, u-x\rangle \geq 0, \\
\langle\beta S(x)+y-x, u-y\rangle \geq 0 \quad \forall u \in K,
\end{gathered}
$$

which is called the system of nonlinear variational inequalities and has been introduced and studied by Verma [26].

For suitable and appropriate choices of the elements $f$ and $g$ and the mappings $S$ and $T$, one can obtain various new and previously known systems of variational inequalities as special cases of the system of generalized parametric nonlinear quasivariational inequalities (2.1). 
We recall the following concept.

Definition 2.1 [1]. Let $M: H \times G \rightarrow 2^{H}$ be such that for each $\lambda \in G, M(\cdot, \lambda): H \rightarrow 2^{H}$ is maximal monotone. Then the implicit resolvent mapping $J_{\rho}^{M(\cdot, \lambda)}$ associated with $M(\cdot, \lambda)$ is defined by $J_{\rho}^{M(\cdot, \lambda)}(u)=(I+\rho M(\cdot, \lambda))^{-1}(u)$ for all $u \in H$, where $\rho>0$ is a constant and $I$ is the identity mapping.

It is known that

$$
\left\|J_{\rho}^{M(\cdot, \lambda)}(x)-J_{\rho}^{M(\cdot, \lambda)}(y)\right\| \leq\|x-y\| \quad \forall x, y \in H .
$$

Definition 2.2. Let $S: H \times G \rightarrow H$ be a mapping.

(1) $S$ is said to be $t$-Lipschitz continuous with respect to the first argument if there exists a constant $t>0$ satisfying

$$
\|S(x, \lambda)-S(y, \lambda)\| \leq t\|x-y\| \quad \forall(x, y, \lambda) \in H \times H \times G .
$$

(2) $S$ is said to be $t$-strongly monotone with respect to the first argument if there exists a constant $t>0$ satisfying

$$
\langle x-y, S(x, \lambda)-S(y, \lambda)\rangle \geq t\|x-y\|^{2} \quad \forall(x, y, \lambda) \in H \times H \times G .
$$

(3) $S$ is said to be $t$-generalized pseudocontractive with respect to the first argument if there exists a constant $t>0$ satisfying

$$
\langle x-y, S(x, \lambda)-S(y, \lambda)\rangle \leq t\|x-y\|^{2} \quad \forall(x, y, \lambda) \in H \times H \times G .
$$

(4) $S$ is said to be $t$-relaxed Lipschitz with respect to the first argument if there exists a constant $t>0$ satisfying

$$
\langle x-y, S(x, \lambda)-S(y, \lambda)\rangle \leq-t\|x-y\|^{2} \quad \forall(x, y, \lambda) \in H \times H \times G .
$$

(5) $S$ is said to be $t$-relaxed monotone with respect to the first argument if there exists a constant $t>0$ satisfying

$$
\langle x-y, S(x, \lambda)-S(y, \lambda)\rangle \geq-t\|x-y\|^{2} \quad \forall(x, y, \lambda) \in H \times H \times G .
$$

LEMMA 2.3 [5]. Let $\left\{a_{n}\right\}_{n \geq 0},\left\{b_{n}\right\}_{n \geq 0},\left\{c_{n}\right\}_{n \geq 0}$, and $\left\{t_{n}\right\}_{n \geq 0}$ be four sequences of nonnegative numbers satisfying

$$
a_{n+1} \leq\left(1-t_{n}\right) a_{n}+t_{n} b_{n}+c_{n} \quad \forall n \geq 0,
$$

where $\left\{t_{n}\right\}_{n \geq 0} \subseteq[0,1], \sum_{n=0}^{\infty} t_{n}=+\infty, \lim _{n \rightarrow \infty} b_{n}=0$, and $\sum_{n=0}^{\infty} c_{n}<\infty$. Then $\lim _{n \rightarrow \infty} a_{n}=0$.

\section{Main results}

The goal of this section is to establish a few existence and uniqueness results as well as sensitivity analysis of solutions for the system of generalized parametric nonlinear quasivariational inequalities (2.1), and prove some convergence results of iterative sequences generated by the algorithm with errors which is based on the following Lemma 3.1. 
1798 Generalized parametric nonlinear quasivariational inequalities

Lemma 3.1. Let $S, T: H \times G \rightarrow H$ be mappings, let $\rho$ and $\beta$ be positive constants, let $f$ and $g$ be arbitrary elements in $H$, and $\lambda \in G$. Then the following statements are equivalent:

(a) the system of generalized parametric nonlinear quasivariational inequalities (2.1) has a solution $(x, y) \in H \times H$;

(b) there exists $(x, y) \in H \times H$ satisfying

$$
\begin{aligned}
& x=J_{\rho}^{M(\cdot, \lambda)}(y-\rho(S(y, \lambda)-T(y, \lambda)-f)), \\
& y=J_{\beta}^{M(\cdot, \lambda)}(x-\beta(S(x, \lambda)-T(x, \lambda)-g))
\end{aligned}
$$

(c) the mapping $F(\cdot, \lambda): H \rightarrow H$ defined by

$$
\begin{aligned}
F(u, \lambda)=J_{\rho}^{M(\cdot, \lambda)}\left[J_{\beta}^{M(\cdot, \lambda)}(u-\beta(S(u, \lambda)-T(u, \lambda)-g))\right. & \\
-\rho\left[S\left(J_{\beta}^{M(\cdot, \lambda)}(u-\beta(S(u, \lambda)-T(u, \lambda)-g)), \lambda\right)\right. & \left.\left.\quad T\left(J_{\beta}^{M(\cdot, \lambda)}(u-\beta(S(u, \lambda)-T(u, \lambda)-g)), \lambda\right)-f\right]\right] \quad \forall u \in H,
\end{aligned}
$$

has a fixed point $x \in H$ and $y=J_{\beta}^{M(\cdot, \lambda)}(x-\beta(S(x, \lambda)-T(x, \lambda)-g))$.

Proof. It is easy to see that

$$
\begin{aligned}
0 \in \rho(S(y, \lambda)-T(y, \lambda)-f)+x-y+\rho M(x, \lambda) \\
\quad \Longleftrightarrow y-\rho(S(y, \lambda)-T(y, \lambda)-f) \in[I+\rho M(\cdot, \lambda)](x) \\
\quad \Longleftrightarrow x=J_{\rho}^{M(\cdot, \lambda)}(y-\rho(S(y, \lambda)-T(y, \lambda)-f)) .
\end{aligned}
$$

Analogously we can obtain that $y=J_{\beta}^{M(\cdot, \lambda)}(x-\beta(S(x, \lambda)-T(x, \lambda)-g))$. That is, (a) $\Leftrightarrow$ (b). Suppose that (c) holds. Obviously, the mapping $F$ has a fixed point $x \in H$ and $y=$ $J_{\beta}^{M(\cdot, \lambda)}(x-\beta(S(x, \lambda)-T(x, \lambda)-g))$. It follows from (3.2) that $x=F(x, \lambda)=J_{\rho}^{M(\cdot, \lambda)}(y-$ $\rho(S(y, \lambda)-T(y, \lambda)-f))$. That is, (3.1) is satisfied, therefore, (b) holds. Conversely, if (b) holds, then (3.1) and (3.2) yield that

$$
\begin{aligned}
x= & J_{\rho}^{M(\cdot, \lambda)}(y-\rho(S(y, \lambda)-T(y, \lambda)-f)) \\
=J_{\rho}^{M(\cdot, \lambda)}\left[J_{\beta}^{M(\cdot, \lambda)}(x-\beta(S(x, \lambda)-T(x, \lambda)-g))\right. & \quad-\rho\left[S\left(J_{\beta}^{M(\cdot, \lambda)}(x-\beta(S(x, \lambda)-T(x, \lambda)-g)), \lambda\right)\right. \\
& \left.\left.\quad-T\left(J_{\beta}^{M(\cdot, \lambda)}(x-\beta(S(x, \lambda)-T(x, \lambda)-g)), \lambda\right)-f\right]\right] \\
= &
\end{aligned}
$$

that is, (c) holds. This completes the proof. 
Remark 3.2. Lemma 2.1 of Nie et al. in [21], [26, Lemma 1.3] of Verma, and [27, Lemma 2.1] of Wu et al. are special cases of Lemma 3.1 in this paper.

Based on Lemma 3.1 we suggest the following general iterative algorithm with errors for the system of generalized parametric nonlinear quasivariational inequalities (2.1).

Algorithm 3.3. For an arbitrarily chosen initial element $x_{0} \in H, \lambda \in G$, compute sequences $\left\{x_{n}\right\}_{n \geq 0}$ and $\left\{y_{n}\right\}_{n \geq 0}$ by the following iterative procedure:

$$
\begin{gathered}
z_{n}=\left(1-b_{n}\right) x_{n}+b_{n} F\left(x_{n}, \lambda\right)+u_{n}, \\
x_{n+1}=\left(1-a_{n}\right) x_{n}+a_{n} F\left(z_{n}, \lambda\right)+v_{n}, \\
y_{n}=J_{\beta}^{M(\cdot, \lambda)}\left(x_{n}-\beta\left(S\left(x_{n}, \lambda\right)-T\left(x_{n}, \lambda\right)-g\right)\right)+w_{n} \quad \forall n \geq 0,
\end{gathered}
$$

where $F$ is defined by (3.2), $\left\{a_{n}\right\}_{n \geq 0}$ and $\left\{b_{n}\right\}_{n \geq 0}$ are any sequences in $[0,1]$, and $\left\{u_{n}\right\}_{n \geq 0}$, $\left\{v_{n}\right\}_{n \geq 0}$, and $\left\{w_{n}\right\}_{n \geq 0}$ are any sequences satisfying

$$
\begin{array}{ll}
\sum_{n=0}^{\infty} a_{n}=+\infty, & \lim _{n \rightarrow \infty}\left\|u_{n}\right\|=0, \\
\sum_{n=0}^{\infty}\left\|v_{n}\right\|<+\infty, & \lim _{n \rightarrow \infty}\left\|w_{n}\right\|=0 .
\end{array}
$$

Theorem 3.4. Assume that $S: H \times G \rightarrow H$ is both s-Lipschitz continuous and a-relaxed monotone with respect to the first argument and $T: H \times G \rightarrow H$ is both $t$-Lipschitz continuous and b-relaxed Lipschitz with respect to the first argument. Suppose that the sequences $\left\{x_{n}\right\}_{n \geq 0}$ and $\left\{y_{n}\right\}_{n \geq 0}$ generated by Algorithm 3.3 satisfy (3.5) and (3.6). If there exist positive constants $\rho$ and $\beta$ satisfying

$$
\max \{\rho, \beta\}<\frac{2(b-a)}{(s+t)^{2}}
$$

then for any given $f, g \in H, \lambda \in G$, the system of generalized parametric nonlinear quasivariational inequalities (2.1) has a unique solution $(x, y) \in H \times H$ and $\lim _{n \rightarrow \infty} x_{n}=x$ and $\lim _{n \rightarrow \infty} y_{n}=y$. Furthermore, if there exists a constant $\eta>0$ such that

$$
\left\|J_{\rho}^{M(\cdot, \lambda)}(w)-J_{\rho}^{M(\cdot, \bar{\lambda})}(w)\right\| \leq \eta\|\lambda-\bar{\lambda}\|
$$

for all $(w, \bar{\lambda}, \lambda) \in H \times G \times G$, and $S$ and $T$ are continuous (resp., uniformly continuous or Lipschitz continuous) with respect to the second argument, then the solutions of the system of generalized parametric nonlinear quasivariational inequalities (2.1) are continuous (resp., uniformly continuous or Lipschitz continuous).

Proof. For each given $\lambda \in G$, we want to prove that $F(\cdot, \lambda): H \rightarrow H$ defined by (3.2) is a contraction mapping. Since $S$ is both $s$-Lipschitz continuous and $a$-relaxed monotone 
1800 Generalized parametric nonlinear quasivariational inequalities

with respect to the first argument, $T$ is both $t$-Lipschitz continuous and $b$-relaxed Lipschitz with respect to the first argument and (2.5), we get that

$$
\begin{aligned}
& \|F(u, \lambda)-F(v, \lambda)\|^{2} \\
& =\| J_{\rho}^{M(\cdot, \lambda)}\left[J_{\beta}^{M(\cdot, \lambda)}(u-\beta(S(u, \lambda)-T(u, \lambda)-g))\right. \\
& -\rho\left[S\left(J_{\beta}^{M(\cdot, \lambda)}(u-\beta(S(u, \lambda)-T(u, \lambda)-g)), \lambda\right)\right. \\
& \left.\left.-T\left(J_{\beta}^{M(\cdot, \lambda)}(u-\beta(S(u, \lambda)-T(u, \lambda)-g)), \lambda\right)-f\right]\right] \\
& -J_{\rho}^{M(\cdot, \lambda)}\left[J_{\beta}^{M(\cdot, \lambda)}(v-\beta(S(\nu, \lambda)-T(v, \lambda)-g))\right. \\
& -\rho\left[S\left(J_{\beta}^{M(\cdot, \lambda)}(v-\beta(S(v, \lambda)-T(v, \lambda)-g)), \lambda\right)\right. \\
& \left.\left.-T\left(J_{\beta}^{M(\cdot, \lambda)}(v-\beta(S(v, \lambda)-T(v, \lambda)-g)), \lambda\right)-f\right]\right]\|\|^{2} \\
& \leq \| J_{\beta}^{M(\cdot, \lambda)}(u-\beta(S(u, \lambda)-T(u, \lambda)-g)) \\
& -J_{\beta}^{M(\cdot, \lambda)}(v-\beta(S(v, \lambda)-T(v, \lambda)-g)) \\
& -\rho\left[S\left(J_{\beta}^{M(\cdot, \lambda)}(u-\beta(S(u, \lambda)-T(u, \lambda)-g)), \lambda\right)\right. \\
& \left.-S\left(J_{\beta}^{M(\cdot, \lambda)}(v-\beta(S(v, \lambda)-T(\nu, \lambda)-g)), \lambda\right)\right] \\
& +\rho\left[T\left(J_{\beta}^{M(\cdot, \lambda)}(u-\beta(S(u, \lambda)-T(u, \lambda)-g)), \lambda\right)\right. \\
& \left.-T\left(J_{\beta}^{M(\cdot, \lambda)}(v-\beta(S(v, \lambda)-T(v, \lambda)-g)), \lambda\right)\right] \|^{2} \\
& =\| J_{\beta}^{M(\cdot, \lambda)}(u-\beta(S(u, \lambda)-T(u, \lambda)-g)) \\
& -J_{\beta}^{M(\cdot, \lambda)}(v-\beta(S(v, \lambda)-T(v, \lambda)-g)) \|^{2} \\
& -2 \rho\left\langle J_{\beta}^{M(\cdot, \lambda)}(u-\beta(S(u, \lambda)-T(u, \lambda)-g))\right. \\
& -J_{\beta}^{M(\cdot, \lambda)}(v-\beta(S(v, \lambda)-T(v, \lambda)-g)), \\
& S\left(J_{\beta}^{M(\cdot, \lambda)}(u-\beta(S(u, \lambda)-T(u, \lambda)-g)), \lambda\right) \\
& \left.-S\left(J_{\beta}^{M(\cdot, \lambda)}(v-\beta(S(v, \lambda)-T(v, \lambda)-g)), \lambda\right)\right\rangle \\
& +2 \rho\left\langle J_{\beta}^{M(\cdot, \lambda)}(u-\beta(S(u, \lambda)-T(u, \lambda)-g))\right. \\
& -J_{\beta}^{M(\cdot, \lambda)}(v-\beta(S(v, \lambda)-T(v, \lambda)-g)), \\
& T\left(J_{\beta}^{M(\cdot, \lambda)}(u-\beta(S(u, \lambda)-T(u, \lambda)-g)), \lambda\right) \\
& \left.-T\left(J_{\beta}^{M(\cdot, \lambda)}(v-\beta(S(v, \lambda)-T(v, \lambda)-g)), \lambda\right)\right\rangle
\end{aligned}
$$


Zeqing Liu et al. 1801

$$
\begin{aligned}
& +\rho^{2} \| S\left(J_{\beta}^{M(\cdot, \lambda)}(u-\beta(S(u, \lambda)-T(u, \lambda)-g)), \lambda\right) \\
& -S\left(J_{\beta}^{M(\cdot, \lambda)}(v-\beta(S(v, \lambda)-T(v, \lambda)-g)), \lambda\right) \\
& -T\left(J_{\beta}^{M(\cdot, \lambda)}(u-\beta(S(u, \lambda)-T(u, \lambda)-g)), \lambda\right) \\
& +T\left(J_{\beta}^{M(\cdot, \lambda)}(v-\beta(S(v, \lambda)-T(v, \lambda)-g)), \lambda\right) \|^{2} \\
& \leq \| J_{\beta}^{M(\cdot, \lambda)}(u-\beta(S(u, \lambda)-T(u, \lambda)-g)) \\
& -J_{\beta}^{M(\cdot, \lambda)}(v-\beta(S(v, \lambda)-T(v, \lambda)-g)) \|^{2} \\
& +2 \rho a \| J_{\beta}^{M(\cdot, \lambda)}(u-\beta(S(u, \lambda)-T(u, \lambda)-g)) \\
& -J_{\beta}^{M(\cdot, \lambda)}(v-\beta(S(v, \lambda)-T(v, \lambda)-g)) \|^{2} \\
& -2 \rho b \| J_{\beta}^{M(\cdot, \lambda)}(u-\beta(S(u, \lambda)-T(u, \lambda)-g)) \\
& -J_{\beta}^{M(\cdot, \lambda)}(v-\beta(S(v, \lambda)-T(v, \lambda)-g)) \|^{2} \\
& +\rho^{2}\left(\| S\left(J_{\beta}^{M(\cdot, \lambda)}(u-\beta(S(u, \lambda)-T(u, \lambda)-g)), \lambda\right)\right. \\
& -S\left(J_{\beta}^{M(\cdot, \lambda)}(v-\beta(S(v, \lambda)-T(v, \lambda)-g)), \lambda\right) \| \\
& +\| T\left(J_{\beta}^{M(\cdot, \lambda)}(u-\beta(S(u, \lambda)-T(u, \lambda)-g)), \lambda\right) \\
& \left.-T\left(J_{\beta}^{M(\cdot, \lambda)}(v-\beta(S(v, \lambda)-T(v, \lambda)-g)), \lambda\right) \|\right)^{2} \\
& \leq\left(1-2(b-a) \rho+(s+t)^{2} \rho^{2}\right) \| J_{\beta}^{M(\cdot, \lambda)}(u-\beta(S(u, \lambda)-T(u, \lambda)-g)) \\
& -J_{\beta}^{M(\cdot, \lambda)}(v-\beta(S(v, \lambda)-T(v, \lambda)-g)) \|^{2} \\
& \leq\left(1-2(b-a) \rho+(s+t)^{2} \rho^{2}\right) \\
& \times\|u-v-\beta[S(u, \lambda)-S(v, \lambda)-T(u, \lambda)+T(v, \lambda)]\|^{2} \\
& =\left(1-2(b-a) \rho+(s+t)^{2} \rho^{2}\right)\left[\|u-v\|^{2}-2 \beta\langle u-v, S(u, \lambda)-S(v, \lambda)\rangle\right. \\
& +2 \beta\langle u-v, T(u, \lambda)-T(v, \lambda)\rangle \\
& \left.+\beta^{2}\|S(u, \lambda)-S(v, \lambda)-T(u, \lambda)+T(v, \lambda)\|^{2}\right] \\
& \leq\left(1-2(b-a) \rho+(s+t)^{2} \rho^{2}\right)\left(1-2(b-a) \beta+(s+t)^{2} \beta^{2}\right)\|u-v\|^{2}
\end{aligned}
$$

for all $u, v \in H$. Put

$$
\theta=\sqrt{1-2(b-a) \rho+(s+t)^{2} \rho^{2}} \cdot \sqrt{1-2(b-a) \beta+(s+t)^{2} \beta^{2}} .
$$


1802 Generalized parametric nonlinear quasivariational inequalities

According to (3.7), we know that $\theta \in(0,1)$. It follows from (3.9) that

$$
\|F(u, \lambda)-F(v, \lambda)\| \leq \theta\|u-v\| \quad \forall u, v \in H .
$$

That is, $F(\cdot, \lambda)$ is a contraction mapping and hence it has a unique fixed point $x \in H$ for each given $\lambda \in G$. Set $y=J_{\beta}^{M(\cdot, \lambda)}(x-\beta(S(x, \lambda)-T(x, \lambda)-g))$. It follows from Lemma 3.1 that the system of generalized parametric nonlinear quasivariational inequalities (2.1) has a solution $(x, y) \in H \times H$.

Now we claim that $(x, y)$ is the unique solution of the system of generalized parametric nonlinear quasivariational inequalities (2.1). In fact, if $(u, v) \in H \times H$ is also a solution of the system of generalized parametric nonlinear quasivariational inequalities $(2.1)$, by Lemma 3.1 we know that $u=F(u, \lambda)$ and $v=J_{\beta}^{M(\cdot, \lambda)}(u-\beta(S(u, \lambda)-T(u, \lambda)-g))$. It follows from the uniqueness of fixed point of $F$ that $u=x$ and hence $v=J_{\beta}^{M(\cdot, \lambda)}(x-$ $\beta(S(x, \lambda)-T(x, \lambda)-g))=y$.

Next we assert that the sequences $\left\{x_{n}\right\}_{n \geq 0}$ and $\left\{y_{n}\right\}_{n \geq 0}$ generated by Algorithm 3.3 converge strongly to $x$ and $y$, respectively. In view of (3.1), (3.5), and (3.11), we conclude that

$$
\begin{aligned}
\left\|z_{n}-x\right\| \leq & \left(1-b_{n}\right)\left\|x_{n}-x\right\|+b_{n}\left\|F\left(x_{n}, \lambda\right)-F(x, \lambda)\right\|+\left\|u_{n}\right\| \\
\leq & \left\|x_{n}-x\right\|+\left\|u_{n}\right\|, \\
\left\|x_{n+1}-x\right\| \leq & \left(1-a_{n}\right)\left\|x_{n}-x\right\|+a_{n} \theta\left\|z_{n}-x\right\|+\left\|v_{n}\right\| \\
\leq & \left(1-(1-\theta) a_{n}\right)\left\|x_{n}-x\right\|+a_{n} \theta\left\|u_{n}\right\|+\left\|v_{n}\right\|, \\
\left\|y_{n}-y\right\|= & \| J_{\beta}^{M(\cdot, \lambda)}\left(x_{n}-\beta\left(S\left(x_{n}, \lambda\right)-T\left(x_{n}, \lambda\right)-g\right)\right) \\
& -J_{\beta}^{M(\cdot, \lambda)}(x-\beta(S(x, \lambda)-T(x, \lambda)-g))\|+\| w_{n} \| \\
\leq & \left\|\left(x_{n}-x\right)-\beta\left(S\left(x_{n}, \lambda\right)-S(x, \lambda)-T\left(x_{n}, \lambda\right)+T(x, \lambda)\right)\right\|+\left\|w_{n}\right\| \\
= & {\left[\left\|x_{n}-x\right\|^{2}-2 \beta\left\langle x_{n}-x, S\left(x_{n}, \lambda\right)-S(x, \lambda)\right\rangle\right.} \\
& +2 \beta\left\langle x_{n}-x, T\left(x_{n}, \lambda\right)-T(x, \lambda)\right\rangle \\
& \left.+\beta^{2}\left\|S\left(x_{n}, \lambda\right)-S(x, \lambda)-T\left(x_{n}, \lambda\right)+T(x, \lambda)\right\|^{2}\right]^{1 / 2}+\left\|w_{n}\right\| \\
\leq & \sqrt{1-2(b-a) \beta+(s+t)^{2} \beta^{2}}\left\|x_{n}-x\right\|+\left\|w_{n}\right\|
\end{aligned}
$$

for all $n \geq 0$, where $F$ and $\theta$ are defined by (3.2) and (3.10), respectively. It follows from Lemma 2.3 and (3.6) that $\lim _{n \rightarrow \infty} x_{n}=x$. Letting $n \rightarrow \infty$ in (3.13), by (3.7) we infer that $\lim _{n \rightarrow \infty} y_{n}=y$. 
Now we analyze the sensitivity of solutions of the generalized parametric nonlinear quasivariational inequalities (2.1). For each given $\lambda \in G$, there exists a unique solution $(x, y) \in H \times H$ denoted by $x(\lambda)$ and $y(\lambda)$ such that (2.1) holds. Hence for each $\lambda, \bar{\lambda} \in G$, we get that

$$
\begin{gathered}
x(\lambda)=F(x(\lambda), \lambda), \quad x(\bar{\lambda})=F(x(\bar{\lambda}), \bar{\lambda}), \\
y(\lambda)=J_{\beta}^{M(\cdot, \lambda)}(x(\lambda)-\beta(S(x, \lambda)-T(x, \lambda)-g)), \\
y(\bar{\lambda})=J_{\beta}^{M(\cdot, \bar{\lambda})}(x(\bar{\lambda})-\beta(S(x, \bar{\lambda})-T(x, \bar{\lambda})-g)), \\
\|x(\lambda)-x(\bar{\lambda})\| \leq\|F(x(\lambda), \lambda)-F(x(\lambda), \bar{\lambda})\|+\|F(x(\lambda), \bar{\lambda})-F(x(\bar{\lambda}), \bar{\lambda})\|, \\
\|y(\lambda)-y(\bar{\lambda})\|=\| J_{\beta}^{M(\cdot, \lambda)}(x(\lambda)-\beta(S(x, \lambda)-T(x, \lambda)-g)) \\
-J_{\beta}^{M(\cdot, \bar{\lambda})}(x(\bar{\lambda})-\beta(S(x, \bar{\lambda})-T(x, \bar{\lambda})-g)) \| .
\end{gathered}
$$

It follows from (2.5) and (3.8) that

$$
\begin{aligned}
& \|F(x(\lambda), \lambda)-F(x(\lambda), \bar{\lambda})\| \\
& \leq \| J_{\rho}^{M(\cdot, \lambda)}\left[J_{\beta}^{M(\cdot, \lambda)}(x(\lambda)-\beta(S(x(\lambda), \lambda)-T(x(\lambda), \lambda)-g))\right. \\
& -\rho\left[S\left(J_{\beta}^{M(\cdot, \lambda)}(x(\lambda)-\beta(S(x(\lambda), \lambda)-T(x(\lambda), \lambda)-g)), \lambda\right)\right. \\
& \left.\left.-T\left(J_{\beta}^{M(\cdot, \lambda)}(x(\lambda)-\beta(S(x(\lambda), \lambda)-T(x(\lambda), \lambda)-g)), \lambda\right)-f\right]\right] \\
& -J_{\rho}^{M(\cdot, \bar{\lambda})}\left[J_{\beta}^{M(\cdot, \lambda)}(x(\lambda)-\beta(S(x(\lambda), \lambda)-T(x(\lambda), \lambda)-g))\right. \\
& -\rho\left[S\left(J_{\beta}^{M(\cdot, \lambda)}(x(\lambda)-\beta(S(x(\lambda), \lambda)-T(x(\lambda), \lambda)-g)), \lambda\right)\right. \\
& \left.\left.-T\left(J_{\beta}^{M(\cdot, \lambda)}(x(\lambda)-\beta(S(x(\lambda), \lambda)-T(x(\lambda), \lambda)-g)), \lambda\right)-f\right]\right] \| \\
& +\| J_{\rho}^{M(\cdot, \bar{\lambda})}\left[J_{\beta}^{M(\cdot, \lambda)}(x(\lambda)-\beta(S(x(\lambda), \lambda)-T(x(\lambda), \lambda)-g))\right. \\
& -\rho\left[S\left(J_{\beta}^{M(\cdot, \lambda)}(x(\lambda)-\beta(S(x(\lambda), \lambda)-T(x(\lambda), \lambda)-g)), \lambda\right)\right. \\
& \left.\left.-T\left(J_{\beta}^{M(\cdot, \lambda)}(x(\lambda)-\beta(S(x(\lambda), \lambda)-T(x(\lambda), \lambda)-g)), \lambda\right)-f\right]\right] \\
& -J_{\rho}^{M(\cdot, \bar{\lambda})}\left[J_{\beta}^{M(\cdot, \bar{\lambda})}(x(\lambda)-\beta(S(x(\lambda), \bar{\lambda})-T(x(\lambda), \bar{\lambda})-g))\right. \\
& -\rho\left[S\left(J_{\beta}^{M(\cdot, \bar{\lambda})}(x(\lambda)-\beta(S(x(\lambda), \bar{\lambda})-T(x(\lambda), \bar{\lambda})-g)), \bar{\lambda}\right)\right. \\
& \left.\left.-T\left(J_{\beta}^{M(\cdot, \bar{\lambda})}(x(\lambda)-\beta(S(x(\lambda), \bar{\lambda})-T(x(\lambda), \bar{\lambda})-g)), \bar{\lambda}\right)-f\right]\right] \| \\
& \leq \eta\|\lambda-\bar{\lambda}\|+\| J_{\beta}^{M(\cdot, \lambda)}(x(\lambda)-\beta(S(x(\lambda), \lambda)-T(x(\lambda), \lambda)-g)) \\
& -J_{\beta}^{M(\cdot, \bar{\lambda})}(x(\lambda)-\beta(S(x(\lambda), \lambda)-T(x(\lambda), \lambda)-g)) \|
\end{aligned}
$$


1804 Generalized parametric nonlinear quasivariational inequalities

$$
\begin{aligned}
& +\| J_{\beta}^{M(\cdot, \bar{\lambda})}(x(\lambda)-\beta(S(x(\lambda), \lambda)-T(x(\lambda), \lambda)-g)) \\
& -J_{\beta}^{M(\cdot, \bar{\lambda})}(x(\lambda)-\beta(S(x(\lambda), \bar{\lambda})-T(x(\lambda), \bar{\lambda})-g)) \| \\
& +\rho\left(\| S\left(J_{\beta}^{M(\cdot, \lambda)}(x(\lambda)-\beta(S(x(\lambda), \lambda)-T(x(\lambda), \lambda)-g)), \lambda\right)\right. \\
& -S\left(J_{\beta}^{M(\cdot, \bar{\lambda})}(x(\lambda)-\beta(S(x(\lambda), \lambda)-T(x(\lambda), \lambda)-g)), \lambda\right) \| \\
& +\| S\left(J_{\beta}^{M(\cdot, \bar{\lambda})}(x(\lambda)-\beta(S(x(\lambda), \lambda)-T(x(\lambda), \lambda)-g)), \lambda\right) \\
& -S\left(J_{\beta}^{M(\cdot, \bar{\lambda})}(x(\lambda)-\beta(S(x(\lambda), \bar{\lambda})-T(x(\lambda), \bar{\lambda})-g)), \lambda\right) \| \\
& +\| S\left(J_{\beta}^{M(\cdot, \bar{\lambda})}(x(\lambda)-\beta(S(x(\lambda), \bar{\lambda})-T(x(\lambda), \bar{\lambda})-g)), \lambda\right) \\
& -S\left(J_{\beta}^{M(\cdot, \bar{\lambda})}(x(\lambda)-\beta(S(x(\lambda), \bar{\lambda})-T(x(\lambda), \bar{\lambda})-g)), \bar{\lambda}\right) \| \\
& +\| T\left(J_{\beta}^{M(\cdot, \lambda)}(x(\lambda)-\beta(S(x(\lambda), \lambda)-T(x(\lambda), \lambda)-g)), \lambda\right) \\
& -T\left(J_{\beta}^{M(\cdot, \bar{\lambda})}(x(\lambda)-\beta(S(x(\lambda), \lambda)-T(x(\lambda), \lambda)-g)), \lambda\right) \| \\
& +\| T\left(J_{\beta}^{M(\cdot, \bar{\lambda})}(x(\lambda)-\beta(S(x(\lambda), \lambda)-T(x(\lambda), \lambda)-g)), \lambda\right) \\
& -T\left(J_{\beta}^{M(\cdot, \bar{\lambda})}(x(\lambda)-\beta(S(x(\lambda), \bar{\lambda})-T(x(\lambda), \bar{\lambda})-g)), \lambda\right) \| \\
& +\| T\left(J_{\beta}^{M(\cdot, \bar{\lambda})}(x(\lambda)-\beta(S(x(\lambda), \bar{\lambda})-T(x(\lambda), \bar{\lambda})-g)), \lambda\right) \\
& \left.-T\left(J_{\beta}^{M(\cdot, \bar{\lambda})}(x(\lambda)-\beta(S(x(\lambda), \bar{\lambda})-T(x(\lambda), \bar{\lambda})-g)), \bar{\lambda}\right) \|\right) \\
& \leq 2 \eta\|\lambda-\bar{\lambda}\|+\beta(\|S(x(\lambda), \lambda)-S(x(\lambda), \bar{\lambda})\| \\
& +\|T(x(\lambda), \lambda)-T(x(\lambda), \bar{\lambda})\|) \\
& +\rho[\eta s\|\lambda-\bar{\lambda}\|+\beta s(\|S(x(\lambda), \lambda)-S(x(\lambda), \bar{\lambda})\|+\|T(x(\lambda), \lambda)-T(x(\lambda), \bar{\lambda})\|) \\
& +\|S(z, \lambda)-S(z, \bar{\lambda})\|+\eta t\|\lambda-\bar{\lambda}\| \\
& +\beta t(\|S(x(\lambda), \lambda)-S(x(\lambda), \bar{\lambda})\|+\|T(x(\lambda), \lambda)-T(x(\lambda), \bar{\lambda})\|) \\
& +\|T(z, \lambda)-T(z, \bar{\lambda})\|] \\
& =[2+(s+t) \rho] \eta\|\lambda-\bar{\lambda}\| \\
& +[1+(s+t) \rho] \beta(\|S(x(\lambda), \lambda)-S(x(\lambda), \bar{\lambda})\|+\|T(x(\lambda), \lambda)-T(x(\lambda), \bar{\lambda})\|) \\
& +\rho(\|S(z, \lambda)-S(z, \bar{\lambda})\|+\|T(z, \lambda)-T(z, \bar{\lambda})\|) \text {, }
\end{aligned}
$$

where $z=J_{\beta}^{M(\cdot, \bar{\lambda})}(x(\lambda)-\beta(S(x(\lambda), \bar{\lambda})-T(x(\lambda), \bar{\lambda})-g))$. It follows from (3.11) that

$$
\|F(x(\lambda), \bar{\lambda})-F(x(\bar{\lambda}), \bar{\lambda})\| \leq \theta\|x(\lambda)-x(\bar{\lambda})\| .
$$


Combining (3.15), (3.17), and (3.18), we infer that

$$
\begin{aligned}
\|x(\lambda)-x(\bar{\lambda})\| & \\
\leq(1-\theta)^{-1}\{[2+(s+t) \rho] \eta\|\lambda-\bar{\lambda}\| & +[1+(s+t) \rho] \beta(\|S(x(\lambda), \lambda)-S(x(\lambda), \bar{\lambda})\| \\
& +\|T(x(\lambda), \lambda)-T(x(\lambda), \bar{\lambda})\|) \\
& +\rho(\|S(z, \lambda)-S(z, \bar{\lambda})\|+\|T(z, \lambda)-T(z, \bar{\lambda})\|)\} .
\end{aligned}
$$

From (3.16), we get that

$$
\begin{aligned}
\| y(\lambda)- & y(\bar{\lambda}) \| \\
\leq & \| J_{\beta}^{M(\cdot, \lambda)}(x(\lambda)-\beta(S(x, \lambda)-T(x, \lambda)-g)) \\
& \quad-J_{\beta}^{M(\cdot, \bar{\lambda})}(x(\lambda)-\beta(S(x, \lambda)-T(x, \lambda)-g)) \| \\
& +\| J_{\beta}^{M(\cdot, \bar{\lambda})}(x(\lambda)-\beta(S(x, \lambda)-T(x, \lambda)-g)) \\
& \quad-J_{\beta}^{M(\cdot, \bar{\lambda})}(x(\bar{\lambda})-\beta(S(x, \bar{\lambda})-T(x, \bar{\lambda})-g)) \| \\
\leq & \eta\|\lambda-\bar{\lambda}\|+\|x(\lambda)-x(\bar{\lambda})\| \\
& +\beta(\|S(x, \lambda)-S(x, \bar{\lambda})\|+\|T(x, \lambda)-T(x, \bar{\lambda})\|) .
\end{aligned}
$$

It follows from (3.19), (3.20), and the continuities of $S$ and $T$ (resp., uniform continuities or Lipschitz continuities) with respect to the second argument that the solutions of the system of generalized parametric nonlinear quasivariational inequalities (2.1) are continuous (resp., uniformly continuous or Lipschitz continuous). This completes the proof.

As in the proof of Theorem 3.4, we get the following result.

Theorem 3.5. Assume that $S: H \times G \rightarrow H$ is both s-Lipschitz continuous and a-strongly monotone with respect to the first argument and $T: H \times G \rightarrow H$ is both $t$-Lipschitz continuous and b-generalized pseudocontractive with respect to the first argument. Suppose that the sequences $\left\{x_{n}\right\}_{n \geq 0}$ and $\left\{y_{n}\right\}_{n \geq 0}$ generated by Algorithm 3.3 satisfy (3.5) and (3.6). If there exist positive constants $\rho$ and $\beta$ satisfying

$$
\max \{\rho, \beta\}<\frac{2(a-b)}{(s+t)^{2}},
$$

then for any given $f, g \in H, \lambda \in G$, the system of generalized parametric nonlinear quasivariational inequalities (2.1) has a unique solution $(x, y) \in H \times H$ and $\lim _{n \rightarrow \infty} x_{n}=x$ and $\lim _{n \rightarrow \infty} y_{n}=y$. Furthermore, if there exists a constant $\eta>0$ satisfying (3.8) and $S$ and $T$ are continuous (resp., uniformly continuous or Lipschitz continuous) with respect to the second argument, then the solutions of the system of generalized parametric nonlinear quasivariational inequalities (2.1) are continuous (resp., uniformly continuous or Lipschitz continuous). 
1806 Generalized parametric nonlinear quasivariational inequalities

Remark 3.6. Theorem 2.1 in [21] is a special case of Theorem 3.5.

\section{Acknowledgment}

This work was supported by the Science Research Foundation of Educational Department of Liaoning Province (2004C063).

\section{References}

[1] R. P. Agarwal, Y. J. Cho, and N. J. Huang, Sensitivity analysis for strongly nonlinear quasivariational inclusions, Appl. Math. Lett. 13 (2000), no. 6, 19-24.

[2] H. Brézis, Opérateurs Maximaux Monotones et Semi-Groupes de Contractions dans les Espaces de Hilbert, North-Holland Mathematics Studies, no. 5. Notas de Matemática (50), NorthHolland, Amsterdam, 1973.

[3] S. Dafermos, Sensitivity analysis in variational inequalities, Math. Oper. Res. 13 (1988), no. 3, 421-434.

[4] H. Dong, B.-S. Lee, and N. J. Huang, Sensitivity analysis for generalized parametric implicit quasivariational inequalities, Nonlinear Anal. Forum 6 (2001), no. 2, 313-320.

[5] L. S. Liu, Ishikawa and Mann iterative process with errors for nonlinear strongly accretive mappings in Banach spaces, J. Math. Anal. Appl. 194 (1995), no. 1, 114-125.

[6] Z. Liu, L. Debnath, S. M. Kang, and J. S. Ume, Completely generalized multivalued nonlinear quasi-variational inclusions, Int. J. Math. Math. Sci. 30 (2002), no. 10, 593-604.

[7] Sensitivity analysis for parametric completely generalized nonlinear implicit quasivariational inclusions, J. Math. Anal. Appl. 277 (2003), no. 1, 142-154.

[8] - Generalized mixed quasivariational inclusions and generalized mixed resolvent equations for fuzzy mappings, Appl. Math. Comput. 149 (2004), no. 3, 879-891.

[9] Z. Liu and S. M. Kang, Comments on the papers involving variational and quasivariational inequalities for fuzzy mappings, Math. Sci. Res. J. 7 (2003), no. 10, 394-339.

[10] - Generalized multivalued nonlinear quasivariational inclusions, Math. Nachr. 253 (2003), 45-54.

[11] Convergence and stability of perturbed three-step iterative algorithm for completely generalized nonlinear quasivariational inequalities, Appl. Math. Comput. 149 (2004), no. 1, 245-258.

[12] Z. Liu, S. M. Kang, and J. S. Ume, On general variational inclusions with noncompact valued mappings, Adv. Nonlinear Var. Inequal. 5 (2002), no. 2, 11-25.

[13]_, Completely generalized multivalued strongly quasivariational inequalities, Publ. Math. Debrecen 62 (2003), no. 1-2, 187-204.

[14]_ Generalized variational inclusions for fuzzy mappings, Adv. Nonlinear Var. Inequal. 6 (2003), no. 1, 31-40.

[15] The solvability of a class of quasivariational inequalities, Adv. Nonlinear Var. Inequal. 6 (2003), no. 2, 69-78.

[16] Z. Liu, J. S. Ume, and S. M. Kang, General strongly nonlinear quasivariational inequalities with relaxed Lipschitz and relaxed monotone mappings, J. Optim. Theory Appl. 114 (2002), no. 3, 639-656.

[17] - Resolvent equations technique for general variational inclusions, Proc. Japan Acad. Ser. A Math. Sci. 78 (2002), no. 10, 188-193.

[18] - Nonlinear variational inequalities on reflexive Banach spaces and topological vector spaces, Int. J. Math. Math. Sci. 2003 (2003), no. 4, 199-207.

[19] Completely generalized quasivariational inequalities, Adv. Nonlinear Var. Inequal. 7 (2004), no. 1, 35-46. 
[20] Z. Liu, L. Wang, S. M. Kang, and J. S. Ume, Sensitivity analysis of solutions for generalized parametric nonlinear variational inequalities, Int. J. Pure Appl. Math. 6 (2003), no. 1, 77-91.

[21] H. Z. Nie, Z. Liu, K. H. Kim, and S. M. Kang, A system of nonlinear variational inequalities involving strongly monotone and pseudocontractive mappings, Adv. Nonlinear Var. Inequal. 6 (2003), no. 2, 91-99.

[22] R. U. Verma, Approximation-solvability of a new system of nonlinear quasivariational inequalities, Adv. Nonlinear Var. Inequal. 3 (2000), no. 1, 47-60.

[23] Computational role of partially relaxed monotone mappings in solvability of a system of nonlinear variational inequalities, Adv. Nonlinear Var. Inequal. 3 (2000), no. 2, 79-86.

[24] - A general iterative algorithm and solvability of nonlinear quasivariational inequalities, Adv. Nonlinear Var. Inequal. 4 (2001), no. 2, 79-87.

[25] - Iterative algorithms and a new system of nonlinear quasivariational inequalities, Adv. Nonlinear Var. Inequal. 4 (2001), no. 1, 117-124.

[26] - Projection methods, algorithms, and a new system of nonlinear variational inequalities, Comput. Math. Appl. 41 (2001), no. 7-8, 1025-1031.

[27] Q. H. Wu, Z. Liu, S. H. Shim, and S. M. Kang, Approximation-solvability of a new system of nonlinear variational inequalities, Math. Sci. Res. J. 7 (2003), no. 8, 338-346.

[28] D. L. Zhu and P. Marcotte, Co-coercivity and its role in the convergence of iterative schemes for solving variational inequalities, SIAM J. Optim. 6 (1996), no. 3, 714-726.

Zeqing Liu: Department of Mathematics, Liaoning Normal University, P.O. Box 200, Dalian, Liaoning 116029, China

E-mail address: zeqingliu@dl.cn

Beibei Zhu: Department of Mathematics, Liaoning Normal University, P.O. Box 200, Dalian, Liaoning 116029, China

E-mail address: beibeizhu81422@hotmail.com

Shin Min Kang: Department of Mathematics, Gyeongsang National University, Chinju 660-701, Korea

E-mail address: smkang@nongae.gsnu.ac.kr

Gwang Il Kim: Department of Mathematics and Research Institute of Natural Science, Gyeongsang National University, Chinju 660-701, Korea

E-mail address: gikim@nongae.gsnu.ac.kr 


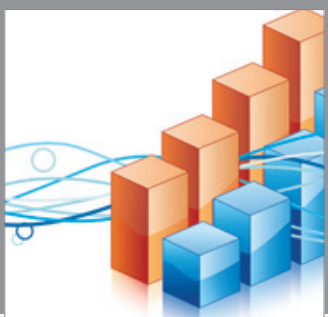

Advances in

Operations Research

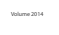

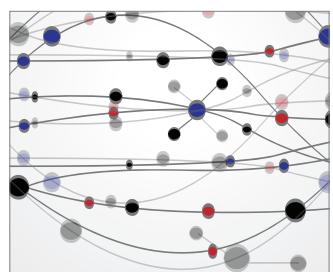

\section{The Scientific} World Journal
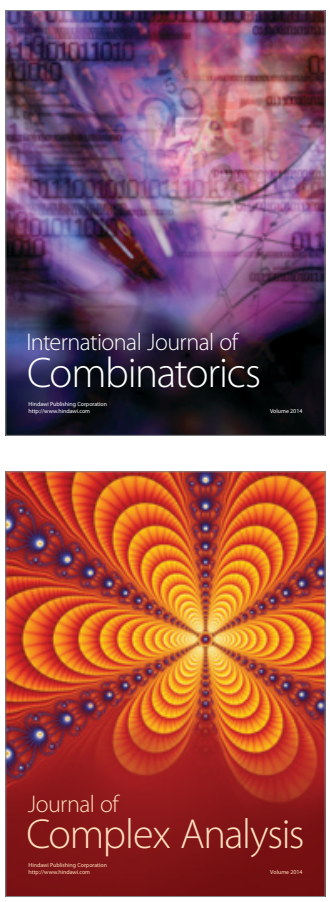

International Journal of

Mathematics and

Mathematical

Sciences
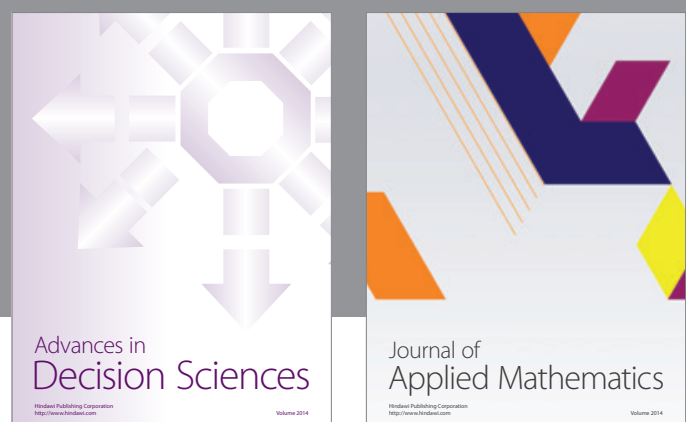

Journal of

Applied Mathematics
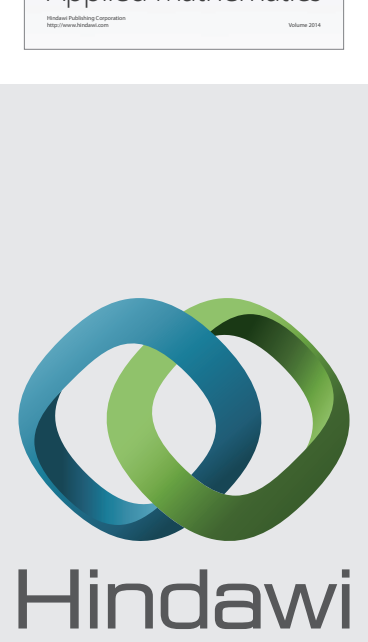

Submit your manuscripts at http://www.hindawi.com
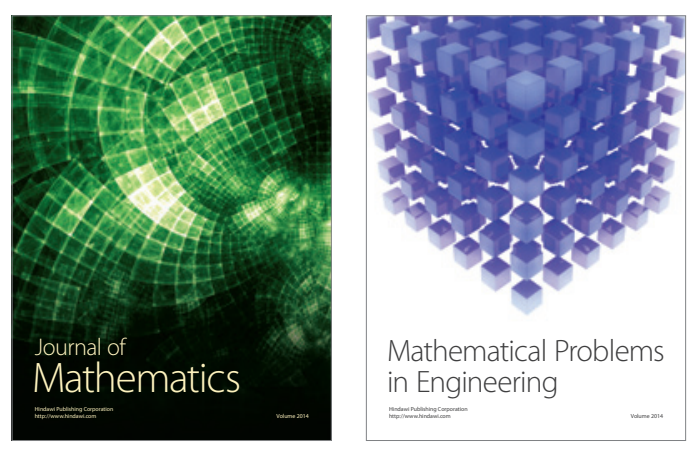

Mathematical Problems in Engineering
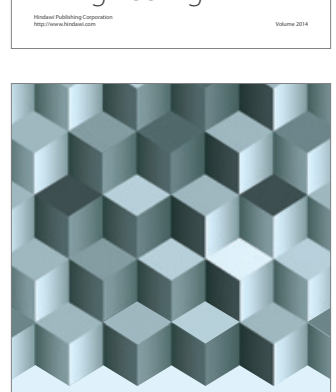

Journal of

Function Spaces
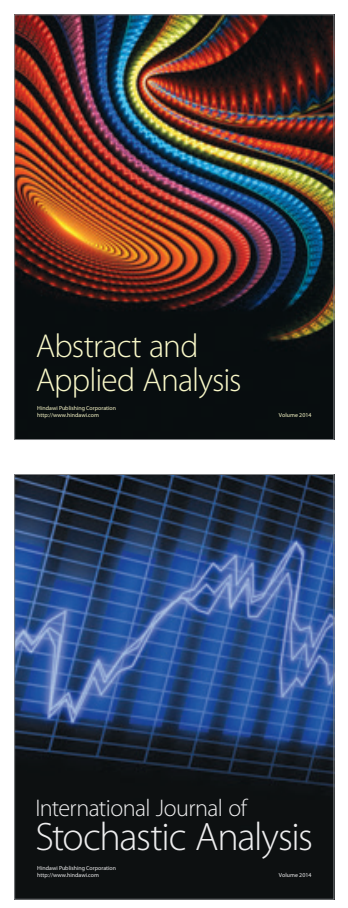

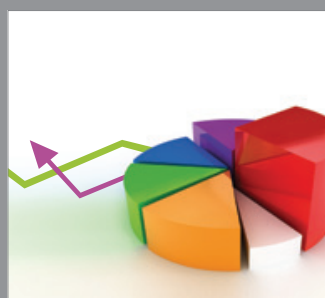

ournal of

Probability and Statistics

Promensencen
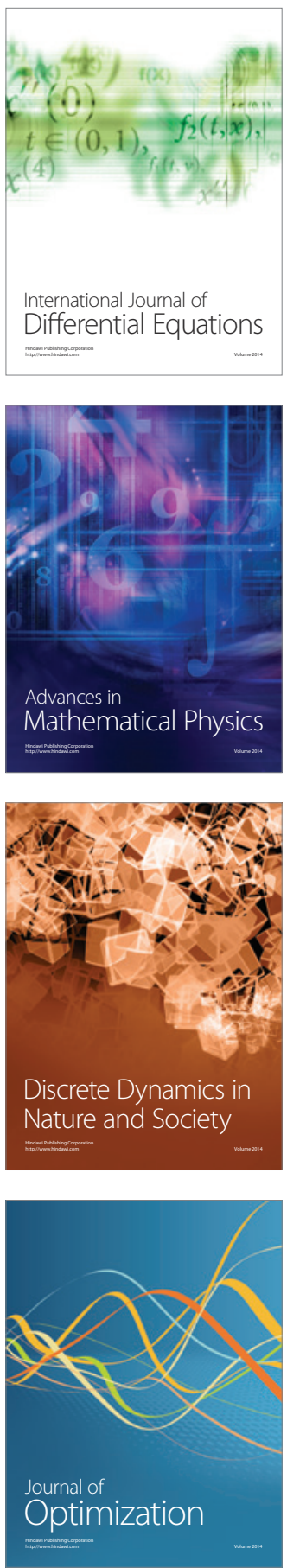